\title{
Quorum sensing modulates colony morphology through alkyl quinolones in Pseudomonas aeruginosa
}

\author{
Rashmi Gupta and Martin Schuster
}

\begin{abstract}
Background: Acyl-homoserine lactone (acyl-HSL) and alkyl quinolone (AQ) based quorum-sensing (QS) systems are important for Pseudomonas aeruginosa virulence and biofilm formation. The effect of QS on biofilm formation is influenced by various genetic and environmental factors. Here, we used a colony biofilm assay to study the effect of the central acyl-HSL QS regulator, LasR, on biofilm formation and structure in the representative clinical $P$. aeruginosa isolate ZK2870.

Results: A lasR mutant exhibited wrinkled colony morphology at $37^{\circ} \mathrm{C}$ in contrast to the smooth colony morphology of the wild-type. Mutational analysis indicated that wrinkling of the lasR mutant is dependent on pel, encoding a biofilm matrix exopolysaccharide. Suppressor mutagenesis and complementation analysis implicated the $\mathrm{AQ}$ signaling pathway as the link between las QS and colony morphology. In this pathway, genes pqsA-D are involved in the synthesis of 4-hydroxyalkyl quinolines ("Series A congeners"), which are converted to 3,4dihydroxyalkyl quinolines ("Series B congeners", including the well-characterized Pseudomonas Quinolone Signal, PQS) by the product of the LasR-dependent pqsH gene. Measurement of $A Q$ in the wild-type, the lasR pqsA::Tn suppressor mutant as well as the defined lasR, pqs H, and lasR pqsH mutants showed a correlation between 4hydroxyalkyl quinoline levels and the degree of colony wrinkling. Most importantly, the las $R$ pqsH double mutant displayed wrinkly morphology without producing any 3,4-dihydroxyalkyl quinolines. Constitutive expression of pqsA-D genes in a lasR pqsR::Tnmutant showed that colony wrinkling does not require the AQ receptor PqsR.

Conclusions: Taken together, these results indicate that the las QS system represses Pel and modulates colony morphology through a 4-hydroxyalkyl quinoline in a PqsR-independent manner, ascribing a novel function to an $\mathrm{AQ}$ other than $\mathrm{PQS}$ in P. aeruginosa.
\end{abstract}

Keywords: Quorum sensing, Pseudomonas aeruginosa, Colony, Alkylquinolone, Acyl-homoserine lactone, Exopolysaccharide, Biofilm

\section{Background}

Pseudomonas aeruginosa is an important opportunistic human pathogen. It is known for its ability to inhabit diverse habitats ranging from soil to immunocompromised individuals [1]. In these environments, it can adopt either a planktonic or a surface-associated biofilm lifestyle. Biofilms, structured surface-associated microbial communities, are of considerable interest as they constitute an important survival strategy in infections

\footnotetext{
* Correspondence: martin.schuster@oregonstate.edu Department of Microbiology, Oregon State University, Corvallis, OR 97331, USA
}

\section{Biomed Central}

[2]. P. aeruginosa forms different types of biofilms depending on the environment. In static liquid culture it forms pellicles at the air-liquid interface, under flow it can form solid surface-associated (SSA) biofilms and on solid agar medium it forms colonies [3]. Colonial growth is an easy and commonly used assay to study development of multicellular structures like biofilms [4-6].

Biofilms are encased in a matrix composed of exopolysaccharide (EPS), but also extracellular DNA (eDNA), proteins, RNA and ions [7]. There are two main EPS in non-mucoid $P$. aeruginosa, Pel (encoded by pelA-G) and Psl (encoded by pslA-O) (Figure 1) [9-11]. Pel is glucose 


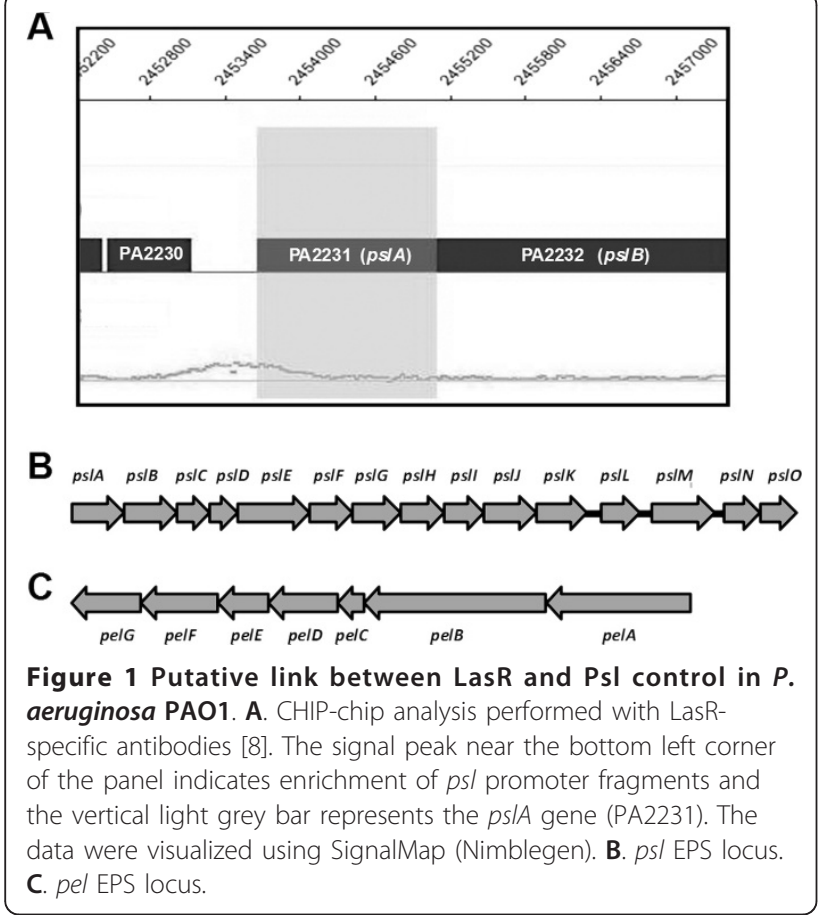

rich whereas Psl is galactose and mannose rich [11-13]. P. aeruginosa strain PA14 only contains pel while strains PAO1 and ZK2870 contain both pel and psl [11,12]. All of these strains are clinical isolates that differ in their aggregative behavior. While strains PA14 and PAO1 are the most commonly used laboratory strains, strain ZK2870 with its autoaggregative phenotype is believed to be the most representative among clinical strains [12].

Quorum sensing (QS) is a cell density-dependent mechanism of bacterial communication that coordinates other group behaviors. $P$. aeruginosa has two complete acyl-homoserine lactone (acyl-HSL)-based QS systems, las and $r h l[14,15]$. They consist of the transcriptional regulators LasR and RhlR and the signal synthases, LasI and RhlI, respectively. LasI and RhlI catalyze the synthesis of N-3-oxododecanoyl-homoserine lactone (3OC12HSL) and N-butryl-homoserine lactone (C4-HSL), which bind and activate their cognate transcriptional regulators LasR and RhlR, respectively. Both systems are arranged in a hierarchical manner with the las system controlling the $r h l$ system $[16,17]$. A third QS system in P. aeruginosa, pqs, is based on alkyl quinolones (AQ) $[18,19]$. This system connects both the las and rhl QS systems. It includes the transcriptional regulator PqsR (MvfR), which positively regulates the expression of the pqsA-E operon. PqsA-D enzymes are involved in the synthesis of 4-hydroxyalkyl quinolines (named Series A congeners by Deziel et al.) [20]. This class of compounds is converted to 3, 4 dihydroxyquinolines (Series B congeners) by a monoxygenase encoded by the $p q s H$ gene [20]. The most prominent Series A congeners are 4-hydroxy-2heptyl quinoline (HHQ) and 4-hydroxy-2-nonyl quinoline (HNQ), and the most prominent Series B congener is 3,4-dihydroxy-2-heptyl quinoline (PQS), due to their established roles as cell-cell signaling molecules. HHQ/ HNQ and PQS bind PqsR with low and high affinity, respectively, and are capable of activating the protein [21-23]. LasR positively regulates AQ production by upregulating pqsR [22] and pqsH [20,24] transcription, although under certain culture conditions, AQ can also be produced in the absence of a functional las system [25]. The $r h l$ system, in turn, represses pqsR and pqsA-E expression [22,26,27]. The AQ biosynthetic enzymes enable $P$. aeruginosa to produce more than 50 distinct AQ molecules $[20,28]$. Together, the three QS systems, las, $r h l$, and $p q s$, regulate $>5 \%$ of the $P$. aeruginosa genome [29-32].

Several studies have investigated the contribution of each QS system to biofilm formation. A functional las system is required for formation of highly structured SSA biofilm communities in $P$. aeruginosa PAO1 [33]. The las system influences biofilm matrix formation and activation of pel EPS [6]. In another study, the las system was shown to indirectly inhibit pel expression through weak activation of the tyrosine phosphatase TpbA [34]. The $r h l$ QS system contributes to maintenance of biofilm architecture through production of rhamnolipid surfactants [35]. The pqs system in turn is implicated in autolysis [36] and maintaining biofilm integrity as a consequence of eDNA release [37]. In addition, the contribution of QS to biofilm formation is modulated by environmental factors such as nutritional cues [38]. Taken together, the role of QS in biofilm formation is multifactorial.

Our recent work suggested yet another connection between QS and EPS production. We showed by chromatin immunoprecipitation-microarray analysis (CHIPchip) and electrophoretic mobility shift assay that LasR binds to the putative promoter region of the Psl EPS operon [8] (Figure 1). This finding led us to investigate in more detail how las $R$ mutation affects EPS production and colony biofilm formation. A lasR mutant of $P$. aeruginosa strain ZK2870 exhibited a pronounced wrinkled colony morphology at $37^{\circ} \mathrm{C}$ suggesting a possible link between las QS and psl expression. However, we found that the wrinkled phenotype is pel rather than psl-dependent. Subsequent suppressor mutagenesis in the lasR mutant background implicated the involvement of the pqs pathway. Phenotypic analysis and quantitation of AQ levels by thin-layer chromatography (TLC) of several QS mutants revealed that a Series A congener, likely other than HHQ or HNQ modulates the structural organization of a colony. This study broadens the 
functional significance of AQ production by $P$. aeruginosa.

\section{Methods}

Bacterial strains and growth conditions

Strains and plasmids are listed in Table 1. We used three strains of $P$. aeruginosa in this study, namely the widely used clinical isolates PAO1 and PA14, and the more recent clinical isolate $\mathrm{ZK} 2870$ (herein abbreviated as $\mathrm{ZK}$ ) [12]. Bacterial cultures were grown at $22^{\circ} \mathrm{C}$ and $37^{\circ} \mathrm{C}$ as specified. Lennox broth (LB) [8] or tryptone broth [12] were used for routine culturing. Tryptic soy broth (TSB) was used for flow-cell biofilm assays. Where appropriate, antibiotics were added to the growth media as follows: Tetracycline and gentamicin, $100 \mu \mathrm{g} / \mathrm{ml}$ for $P$. aeruginosa and $10 \mu \mathrm{g} / \mathrm{ml}$ for Escherichia coli; carbenicillin, $200 \mu \mathrm{g} / \mathrm{ml}$ for $P$. aeruginosa; ampicillin, $100 \mu \mathrm{g} / \mathrm{ml}$ for $E$. coli.

\section{Strain and plasmid constructions}

Deletion mutants were constructed using the strategy of Hoang et al. [45]. ZK lasR and lasI mutants were generated by introducing the previously constructed allelic

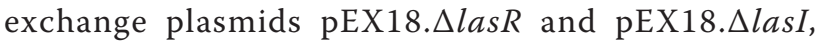
respectively [41], into the parent strain and selecting on LB agar containing nalidixic acid $(20 \mu \mathrm{g} / \mathrm{ml})$ and tetracycline. Double cross-over recombinants were further selected on LB plates supplemented with $5 \%$ sucrose [45]. The $p q s H$ and $t b p A$ in-frame deletions were constructed using SOE-PCR [48]. The respective primers are listed in Additional file 1: Table S1. The deletion

Table 1 Strains and plasmids

\begin{tabular}{|c|c|c|}
\hline $\begin{array}{l}\text { Strain or } \\
\text { plasmid } \\
\text { Strain } \\
\end{array}$ & Relevant property & Reference \\
\hline \multicolumn{3}{|l|}{$P$. aeruginosa } \\
\hline PA14 & Wild-type & [39] \\
\hline PAO1 & Wild-type & {$[40]$} \\
\hline ZK2870 & Wild-type & {$[12]$} \\
\hline PAO1 lasR & Markerless lasR mutant derived from PAO1 & [41] \\
\hline PA14 lasR & TnphoA lasR mutant derived from PA14 & {$[42]$} \\
\hline ZK lasR & Markerless in-frame lasR deletion in ZK2870 & This study \\
\hline ZK pelA & Markerless pelA deletion in ZK2870 & [11] \\
\hline ZK psID & Markerless psID deletion in ZK2870 & {$[11]$} \\
\hline ZK lasl & Markerless las/ deletion in ZK2870 & This study \\
\hline ZK pelA lasR & Markerless lasR deletion in a pelA mutant of ZK2870 & This study \\
\hline ZK psID lasR & Markerless lasR deletion in a psID mutant of ZK2870 & This study \\
\hline ZK pqsH & Markerless pqsH deletion in ZK2870 & This study \\
\hline ZK tpbA & Markerless pqsH deletion in ZK2870 & This study \\
\hline ZK lasR & pqsA suppressor mutation in a lasR mutant of ZK2870 & This study \\
\hline \multicolumn{3}{|l|}{ pqsA::Tn } \\
\hline ZK lasR & pqsR suppressor mutation in a lasR mutant of ZK2870 & This study \\
\hline \multicolumn{3}{|l|}{ pqsR::Tn } \\
\hline \multicolumn{3}{|l|}{ E. coli } \\
\hline $\mathrm{DH} 5 \alpha$ & $\mathrm{F}^{-} \phi 80 \mathrm{~d} / a c Z \Delta \mathrm{M} 15 \Delta\left(\right.$ lacZYA-argF) U169 deoR recA1 endA1 hsdR17( $\left.\mathrm{r}_{\mathrm{K}}^{-} \mathrm{m}_{\mathrm{K}}^{+}{ }^{+}\right)$phoA supE44 $\lambda^{-}$thi-1 gyrA96 relA1 & Invitrogen \\
\hline SM10 & Mobilizing strain, RP4 tra genes integrated in the chromosome, thi-1 thr leu tonA lacY supE recA::RP4-2-Tc::Mu Km ${ }^{r}$ & [43] \\
\hline S17-1/ג pir & $\mathrm{Tp}^{R} \mathrm{Sm}^{\mathrm{R}}$ recA, thi, pro, hsdR-M+RP4: 2-Tc:Mu: Km Tn7 $\lambda$ pir & [43] \\
\hline \multicolumn{3}{|l|}{ Plasmid } \\
\hline mini-CTX-lacZ & Chromosomal integration vector, $T c^{R}$ & [44] \\
\hline pEX18 Tc & Allelic exchange suicide vector, $T c^{R}$ & [45] \\
\hline pEX18. AlasR & Suicide vector with lasR in-frame deletion & [41] \\
\hline pEX18. lasl & Suicide vector with lasl in-frame deletion & {$[41]$} \\
\hline $\mathrm{pEX} 18 . \Delta t p b A$ & Suicide vector containing tpbA in-frame deletion & This study \\
\hline pLM1 & Tn5 delivery vector, $\mathrm{Gm}^{\mathrm{R}}$ & {$[46]$} \\
\hline pLG10 & pqsA-E operon cloned in pUCP18, $A p^{R}$ & {$[24]$} \\
\hline pRG10 & pqsA-D operon cloned under control of $\mathrm{P}_{\text {lac }}$ of $\mathrm{pUCP} 18, \mathrm{Ap}^{\mathrm{R}}$ & This study \\
\hline pRG11 & Promoter region of pel cloned in mini-CTX-lacZ vector & This study \\
\hline pUCP18 & Parent vector of pLG10, $A p^{R}$ & {$[47]$} \\
\hline
\end{tabular}


constructs obtained from SOE-PCR were digested with the appropriate restriction enzymes (see Additional file 1: Table S1) and ligated into equally digested pEX8 [45]. The resulting constructs pEX18. $\Delta p q s H$ and pEX18. $\triangle t p b A$ were transformed into $E$. coli SM10. Mating with $P$. aeruginosa $\mathrm{ZK}$ and appropriate selection as discussed above yielded $p q s H$ and $t p b A$ deletion mutants. The pelA las $R$ and $p s l D$ las $R$ double mutants were constructed by generating an in-frame las $R$ deletion (as described above) in pelA and pslD mutant backgrounds, respectively. A lasR pqs $H$ double mutant was constructed by $p q s H$ deletion in a lasR mutant background. Proper construction of deletion mutants was confirmed by PCR amplification of chromosomal DNA. The plasmid pRG10 was constructed by amplifying a $5.5 \mathrm{~kb}$ region containing the pqsA-D genes using appropriate primers (see Additional file 1: Table S1) and cloning between the PstI and HindIII restriction sites of the pUCP18 vector [47].

\section{Colony biofilm assay}

Bacterial cultures were grown overnight in $\mathrm{LB}$ at $37^{\circ} \mathrm{C}$. The overnight culture was diluted to an optical density $\left(\mathrm{OD}_{600}\right)$ of 0.0025 in tryptone broth and $10 \mu \mathrm{l}$ of the diluted culture was spotted onto Congo red plates [12]. The Congo red medium contained tryptone $(10 \mathrm{~g} / \mathrm{l})$, granulated agar $(0.5 \%)$, Congo red $(40 \mathrm{mg} / \mathrm{l})$, and Coomassie brilliant blue R 250 ( $20 \mathrm{mg} / \mathrm{l})$. The plates were wrapped with aluminum foil and incubated at $37^{\circ} \mathrm{C}$ for 3-5 days. For bacterial strains containing plasmid pLG10 or pRG10, carbenicillin was added to the medium.

\section{Chemical supplementation}

3OC12-HSL was added to Congo red plates buffered with $50 \mu \mathrm{M}$ 3-(N-morpholino) propanesulfonic acid (MOPS) at a final concentration of $10 \mu \mathrm{M}$. Pyocyanin was added to Congo red plates at a final concentration of $50 \mu \mathrm{M}$. HHQ (a gift from M. whitelely, University of Texas) and HNQ (a gift from P. Williams, University of Nottingham) were added to MOPS-buffered Congo red plates at a final concentration of $50 \mu \mathrm{M}$ or directly to the bacterial inoculum at final concentrations of 20, 100 and $500 \mu \mathrm{M}$. The respective solvents ethyl acetate, dimethyl sulfoxide (DMSO), and methanol were used as controls.

\section{Pel'-lacZ-reporter construction and $\beta$-galactosidase measurements}

A $555 \mathrm{bp}$ promoter region of the pel operon was amplified from the ZK strain using the primers listed in Additional file 1: Table S1 and cloned upstream of the lacZ gene in the integration vector mini-CTX-lac $Z$ [44]. The resulting plasmid pRG11 was then inserted into the chromosome of the wild-type and the las $R$ mutant as described [44]. As a control, the mini-CTX-lacZ parent vector was also integrated into the genome. The colonies of the ZK wild-type and the lasR mutant grown on Congo red plates at $37^{\circ} \mathrm{C}$ were used to measure $\beta$ galactosidase levels. A colony was cut out on the $3^{\text {rd }}$, $4^{\text {th }}$, and the $5^{\text {th }}$ day and suspended in $2 \mathrm{ml}$ of $50 \mathrm{mM}$ phosphate buffer, $\mathrm{pH} 7.4$, in a $15 \mathrm{ml}$ conical tube. Cells were lysed by sonication. The total protein was estimated by Bradford assay [49]. The sonicated sample was centrifuged at $4^{\circ} \mathrm{C}$ for $30 \mathrm{~min}$. The resulting supernatant was used to measure $\beta$-galactosidase activity as described previously [50].

\section{Pellicle biofilm assay}

Cultures were inoculated in tryptone broth at an $\mathrm{OD}_{600}$ of 0.0025 and incubated at $22^{\circ} \mathrm{C}$ and $37^{\circ} \mathrm{C}$ without shaking [11]. After 24, 48 and $72 \mathrm{~h}$, pellicle formation was observed at the air-liquid interface.

\section{Microtiter plate biofilm assay}

Biofilm formation in a microtiter format was assayed as described [11]. Overnight cultures of the wild-type and the las $R$ mutant grown in $\mathrm{LB}$ broth at $37^{\circ} \mathrm{C}$ were diluted 1:100 in tryptone broth. One hundred and fifty $\mu \mathrm{l}$ of the diluted culture was added to 96-well polystyrene microtiter plates (Cellstar-Greiner Bio-one) and incubated at $22^{\circ} \mathrm{C}$ and $37^{\circ} \mathrm{C}$ without shaking for 48 and $72 \mathrm{~h}$. Microtiter plates were rinsed in running hot water. Adherent cells were then stained with $1 \%$ crystal violet for $20 \mathrm{~min}$. The microtiter plate was again rinsed in running hot water. Ethanol was added to each dry well and the samples were allowed to stand for $20 \mathrm{~min}$. Absorbance was measured at $590 \mathrm{~nm}$.

\section{Flow-cell biofilm assay}

Biofilms were grown at $37^{\circ} \mathrm{C}$ in flow chambers. The system was assembled as described [33,51]. The cultures for inoculation were prepared from mid-exponential phase $\left(\mathrm{OD}_{600}\right.$ of $\left.0.4-0.8\right) \mathrm{TSB}$ cultures grown at $37^{\circ} \mathrm{C}$. The cultures were diluted to an $\mathrm{OD}_{600}$ of 0.05 in 1:100 diluted TSB medium and injected into the flow cell. Flow was initiated after $1 \mathrm{~h}$. The diluted TSB was supplied at a flow rate of $180 \mu \mathrm{l} / \mathrm{min}$ using a peristaltic pump (Watson Marlow 205S). Images were taken when biofilms had matured (day 3) with an inverted Zeiss LSM Zeta 510 confocal laser scanning microscope (CLSM) using a 63X oil immersion lens. The manufacturer's software and Adobe Photoshop were used for image processing.

\section{Suppressor mutagenesis}

For transposon mutagenesis, biparental matings were set up between the $E$. coli donor (S17-1- $\lambda$ pir/pLM1) and the $P$. aeruginosa recipient strain (ZK las $R$ mutant) as described [52]. The suicide plasmid pLM1 carries a miniTn 5 transposon. The transposon insertion mutants 
were selected on LB agar plates containing gentamicin $(30 \mu \mathrm{g} / \mathrm{ml})$ and nalidixic acid $(20 \mu \mathrm{g} / \mathrm{ml})$. Colonies were picked manually and patched onto rectangular LB plates containing gentamicin $(30 \mu \mathrm{g} / \mathrm{ml})$ in a 96 -well format. Plates were incubated at $37^{\circ} \mathrm{C}$ for one day and then replica-plated onto rectangular Congo red plates using a 96-well-pin replicator. The ZK wild-type and the lasR mutant were included as controls. These plates were incubated for $3-5$ days at $37^{\circ} \mathrm{C}$. Candidate revertants exhibiting a smooth colony morphology identical to the wild-type were streaked for isolated colonies and subjected to a second screen. This screen involved performing the original colony biofilm assay as described earlier. Mutants which again showed a smooth phenotype were considered to be true revertants.

\section{Mapping of transposon insertions}

Genomic DNA was isolated from the selected transposon mutants (Qiagen PUREGENE kit) and was digested with $\mathrm{Ncol}$. The transposon does not contain an $\mathrm{NcoI}$ restriction site and has an R6K origin of replication. The digested DNA was self-ligated with T4 DNA ligase (New England Biolabs) and electroporated into chemically competent $E$. coli S17-1/ $\lambda$ pir [43]. Plasmid DNA was isolated from gentamicin-resistant colonies and was sequenced using the Tn5 specific primer tnpRL17-1 [53]. Transposon insertions were mapped by comparing sequences to a Pseudomonas protein database using BlastX.

\section{Overexpression of pqsA-E}

The appropriate strains were transformed with plasmid pLG10 [24] and pRG10 carrying the pqsA-E operon and pqs $A-D$ operon under the control of native and constitutive promoters, respectively, or with pUCP18 [47], the parent vector from which pLG10 and pRG10 were derived.

\section{Thin-layer chromatography (TLC)}

Samples for TLC analysis were prepared from 3-5 dayold colonies. Two colonies of each strain grown on the same plate were cut out from the agar with minimum possible agar contamination. One colony was used for total protein estimation and the other for AQ extraction. Total protein was estimated by Bradford assay [49] as described earlier for $\beta$-galactosidase measurements. For AQ extraction, a colony was harvested and suspended in $5 \mathrm{ml}$ methanol, homogenized with a tissue tearor, and allowed to stand for $10 \mathrm{~min}$. The suspension was centrifuged for $30 \mathrm{~min}$ at 4000 r.p.m. at $4^{\circ} \mathrm{C}$. The supernatant was filtered through a $0.2 \mu \mathrm{M}$ syringe filter and the filtrate was collected in glass vials prewashed with acetone. The samples were then airdried, reconstituted in $500 \mu \mathrm{l}$ of methanol and transferred to $2 \mathrm{ml}$ glass vials. They were again air-dried and finally reconstituted in $100 \mu \mathrm{l}$ of methanol. TLC plates were prepared and samples were run as described [23]. Five $\mu$ lof the sample (normalized to total protein), $2 \mu \mathrm{l}$ of the standards-PQS (5 and 10 $\mathrm{mM}$ ), and HHQ (2.5 and $5 \mathrm{mM}$ ) ) were used. AQ levels were estimated in the wild-type and the las $R$ mutant by densitometric analysis of relative spot intensities using Imagequant TL software (GE Healthcare) from two independent experiments.

\section{Results and discussion}

\section{A ZK lasR mutant forms wrinkly colonies}

We investigated the effect of a lasR mutation on colony morphology as an indicator of matrix production $[6,12]$. A wrinkled colony phenotype is generally associated with increased EPS production and biofilm formation. Our agar medium also contained Congo-red, which may stain colonies overproducing EPS [54], but is not always a reliable indicator, especially at $37^{\circ} \mathrm{C}$ [5]. We therefore focused on colony wrinkling (rugosity). We grew the wild-type and lasR mutants of three $P$. aeruginosa strains, namely widely used strains PAO1 and PA14, and the autoaggregative strain ZK2870 [12], on agar plates for 5 days at $37^{\circ} \mathrm{C}$ and at $22^{\circ} \mathrm{C}$. Growth conditions are identical to those previously used to investigate EPS-dependent colony morphology $[6,12]$. We did not observe any significant differences in rugosity between the PAO1 wild-type and las $R$ mutant strains at either temperature (Figure $2 \mathrm{~A})$. However, the colonies of the wild-type and the lasR mutant of strains PA14 and ZK showed striking differences. A PA14 lasR mutant formed a flat, smooth colony as compared to the wrinkled wild-type phenotype at $22^{\circ} \mathrm{C}$ (Figure 2A). On the contrary, a ZK lasR mutant formed a distinctive wrinkled colony at $37^{\circ} \mathrm{C}$ while the wild-type formed a smooth colony (Figure 2A). At room temperature, the morphological difference between the wild-type and the ZK lasR mutant was not as pronounced. A positive regulatory link between las QS, pel transcription and colony morphology has already been described in strain PA14, which only carries Pel EPS [6]. The apparently reverse relationship between las QS and colony morphology at $37^{\circ} \mathrm{C}$ in strain ZK, which harbors both Pel and Psl, was intriguing to us and is the focus of this study.

To confirm that the observed phenotype is generally dependent on a non-functional las system, we also constructed a ZK lasI in-frame deletion mutant. A ZK lasI mutant showed a well defined wrinkled colony like the lasR mutant at $37^{\circ} \mathrm{C}$ (Figure 2B). Supplementation of the lasI mutation with exogenous 3OC12-HSL signal virtually restored the smooth wild-type phenotype (Figure $2 \mathrm{~B}$ ). This confirms that the las system is responsible for the wrinkled colony phenotype. We used the ZK lasR mutant for further study. 


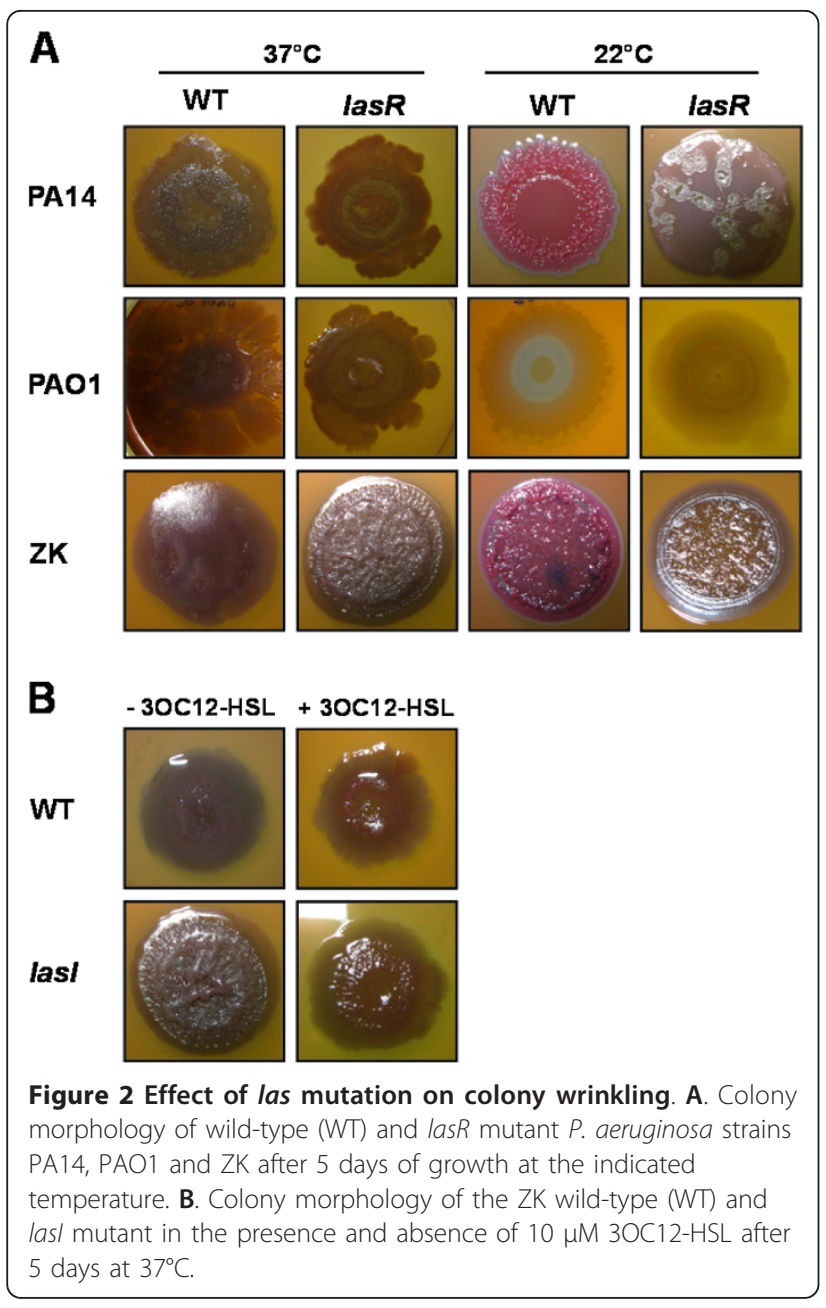

Genetic analysis indicates involvement of pel rather than psl We performed mutational analysis to investigate whether Pel or Psl EPS might cause wrinkling of the lasR mutant. We constructed pelA lasR and pslD lasR double mutants and compared their colony morphology to that of the las $R$ mutant and the wild-type parent. A pelA lasR double mutant showed a nearly smooth colony phenotype while the pslD lasR mutant showed a wrinkled phenotype like the lasR mutant (Figure 3). We evaluated the contribution of pel alone by comparing the colony morphology of a pelA mutant to the wild-type. The pelA colony phenotype was indistinguishable to that of the wild-type. The partial loss of wrinkles in a pelA lasR double mutant therefore indicates inhibition of Pel by LasR.

To determine whether inhibition is at the transcriptional level, we measured pelA transcription in the wildtype and the lasR mutant using a pelA'-lacZ transcriptional fusion inserted at a neutral chromosomal site. We harvested colonies after 3, 4 and 5 days, because a ZK lasR mutant begins to show wrinkling at day 3 . We found no difference in pelA transcription in the wild-

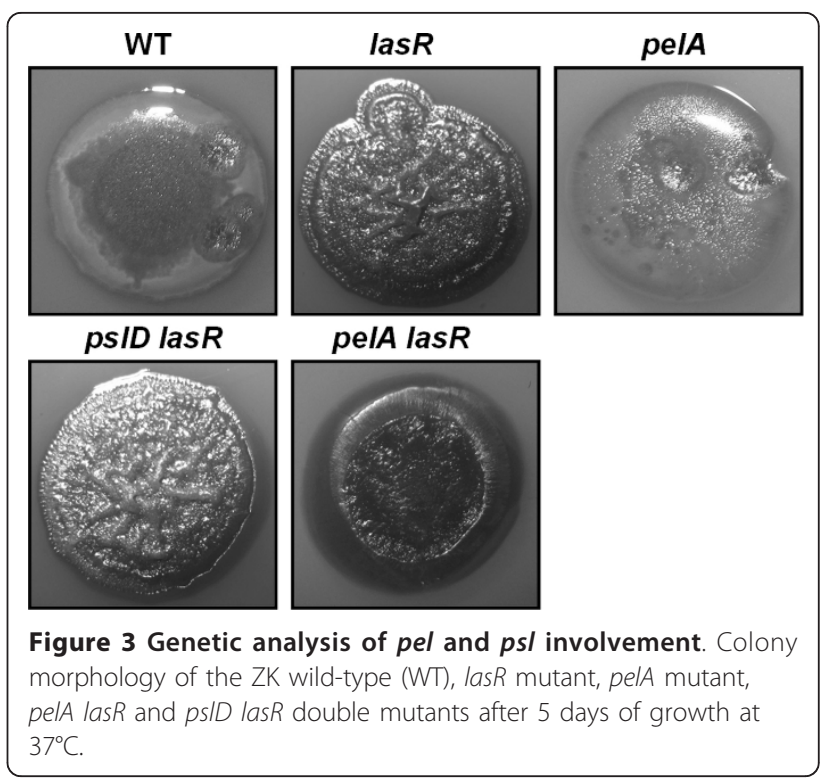

type and the lasR mutant (data not shown). This indicates that pel regulation is at the posttranscriptional level. We attempted to investigate this possibility by quantifying EPS; however, we were unable to perform an EPS composition and linkage analysis because of insufficient amounts of purified EPS extracted from colonies required for such analysis.

\section{Investigation of other factors associated with pel and the} wrinkled colony phenotype

We investigated the role of phenazines and of the tyrosine phosphatase TpbA in the observed wrinkled phenotype of a ZK lasR mutant as both modulate structural organization of P. aeruginosa PA14 colony biofilms [34,55]. We examined the relationship between phenazine deficiency and the wrinkled phenotype through addition of pyocyanin to the agar medium. Pyocyanin supplementation did not result in loss of wrinkles in the lasR mutant (Figure 4A). Inhibition of TpbA in strain PA14 has been shown to enhance pel expression at $37^{\circ} \mathrm{C}$, resulting in a wrinkled colony phenotype [34]. We therefore constructed a $t p b A$ mutant in the ZK background and examined colony morphology. The tpbA mutant remained as smooth as the wild-type (Figure 4B). These results indicate neither pyocyanin nor TpbA are responsible for the wrinkled phenotype of the ZK lasR mutant.

\section{Hydrated lasR mutant biofilms do not show altered architecture}

The involvement of pel in the wrinkled colony morphology of the ZK lasR mutant suggested that it might exhibit generally altered biofilm architecture. We investigated pellicle formation of standing cultures as well as biofilm formation in microtiter plates and flow- 


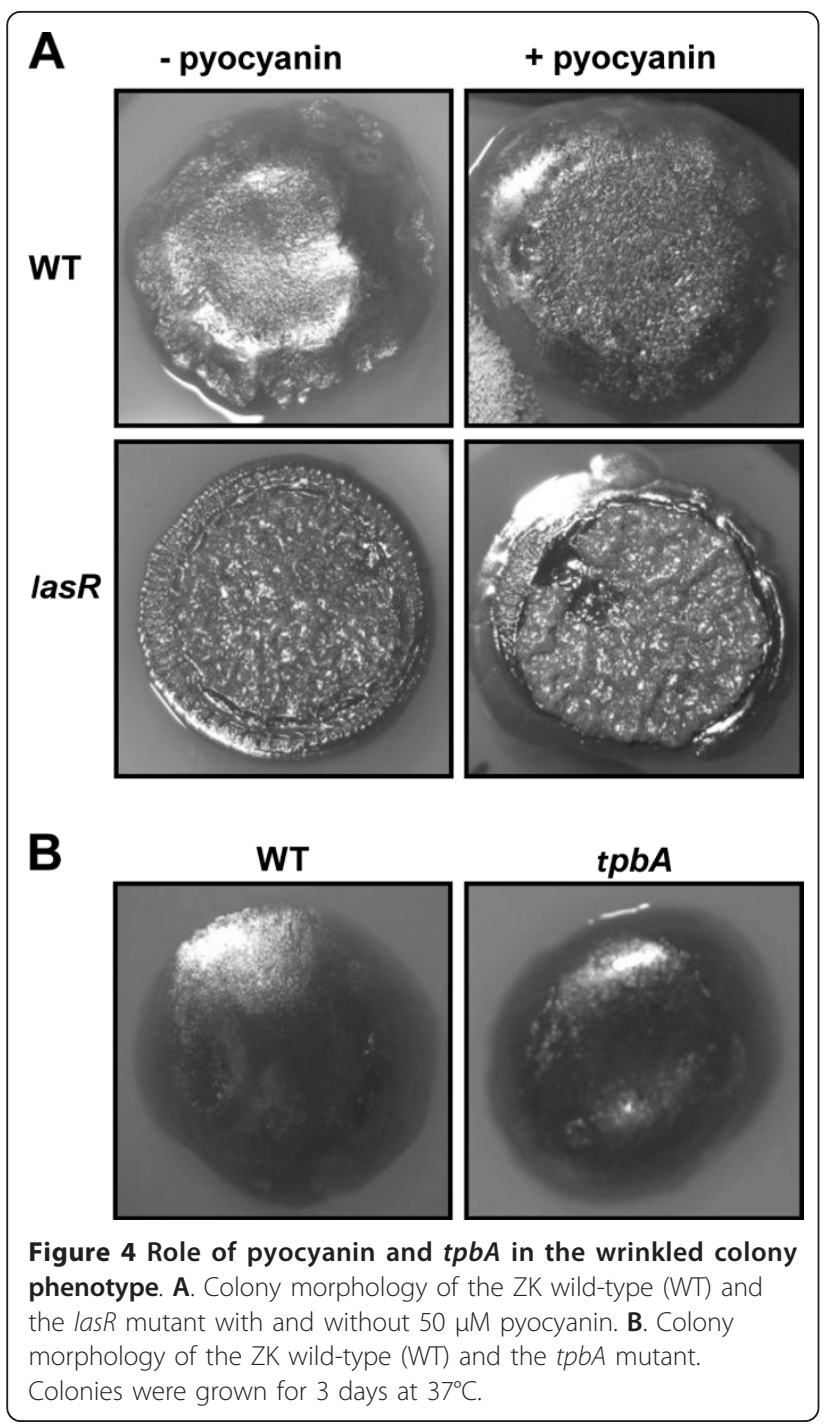

cells. Flow-cell biofilms of the wild-type and the lasR mutant after 3 days of growth are shown in Figure 5. Neither assay revealed any differences between the two strains. This is consistent with recent results by Colvin et al., who also found no defect in attachment or biofilm development for a pel mutant of strain PAO1 [56]. There is a difference in the degree of hydration in the three biofilm assays we employed. Submerged flow-cell biofilms are fully saturated and hydrated, pellicles and microtiter plate biofilms that form at the air-liquid interface are somewhat less hydrated, whereas colonies on agar are the least hydrated [57]. It is possible that the observed phenotype only manifests under conditions of low hydration.

\section{Suppressor mutagenesis implicates the pqs pathway}

Transposon mutagenesis was performed in the ZK lasR mutant background to identify the regulatory link between the las QS system and colony morphology. Around 10,000 mutants were screened for reversion to a smooth phenotype. We identified 38 mutants, and mapped transposon insertions in 25 (Additional file 2: Table S2). We found 9 transposon insertions in the pqsA-D genes of the AQ biosynthesis operon and one insertion in the gene encoding the transcriptional regulator PqsR that activates $p q s A-E$ expression (Figure 6). Given the large fraction of hits (10 out of 25 or $40 \%$ ), the role of the $p q s$ operon was apparent even without mapping the remaining transposon mutants. We did not identify any insertions in $p q s H$, which promotes the conversion of Series A (4-hydroxyalkyl quinolines) to Series B (3,4 dihydroxyalkyl quinolines) congeners nor in $p q s E$, which encodes a putative global regulator $[20,58]$. Surprisingly, we also did not identify a transposon insertion in the pel operon, although our data in Figure 3 show that the lasR pel mutant forms a smooth colony. We found that this mutant displayed very slight wrinkling under the conditions employed for the high throughput screen, in which our primary focus was on the identification of the most obvious smooth revertants. The wrinkly phenotype of the lasR pqsA::Tn suppressor mutant could be restored by introducing plasmid pLG10 [24], which expresses the $p q s A-E$ operon from its native promoter (Figure 7A). This verifies that the products of this operon are indeed responsible for the wrinkled phenotype of the las $R$ pqsA:Tn mutant. To investigate whether $p q s A-D$ dependent wrinkling of the lasR mutant is through PqsR, we introduced plasmid pRG10 into the lasR pqsR:Tn mutant. This plasmid constitutively expresses the pqsA-D operon from a lac promoter. The lasR pqsR:Tn mutant colony was as wrinkly as that of the las $R$ mutant indicating that this phenotype is independent of PqsR (Figure 7B).

\section{A Series A AQ congener causes the wrinkled phenotype}

The previous finding that las $R$ mutants overproduce Series A congeners $[20,59]$ and the fact that we did not find any insertion in the $p q s H$ gene indicate that Series A congeners rather than Series B congeners are responsible for the wrinkled phenotype. We therefore examined this notion further by correlating colony morphology and AQ production, as measured by TLC, in a number of mutant strains. TLC allowed us to distinguish between high-abundance Series A and B congeners. This assay was developed and has been optimized to detect $\mathrm{PQS}$ and $\mathrm{HHQ}$, owing to their important roles in cell-cell signaling. Compounds within each series, especially $\mathrm{C} 7$ and $\mathrm{C} 9$ congeners, are not well separated, and low-abundance compounds may not be detectable [23]. We included the wild-type, the lasR mutant, and the lasR pqsA::Tn suppressor mutant in this analysis. In addition, we constructed a $p q s H$ single mutant and a 

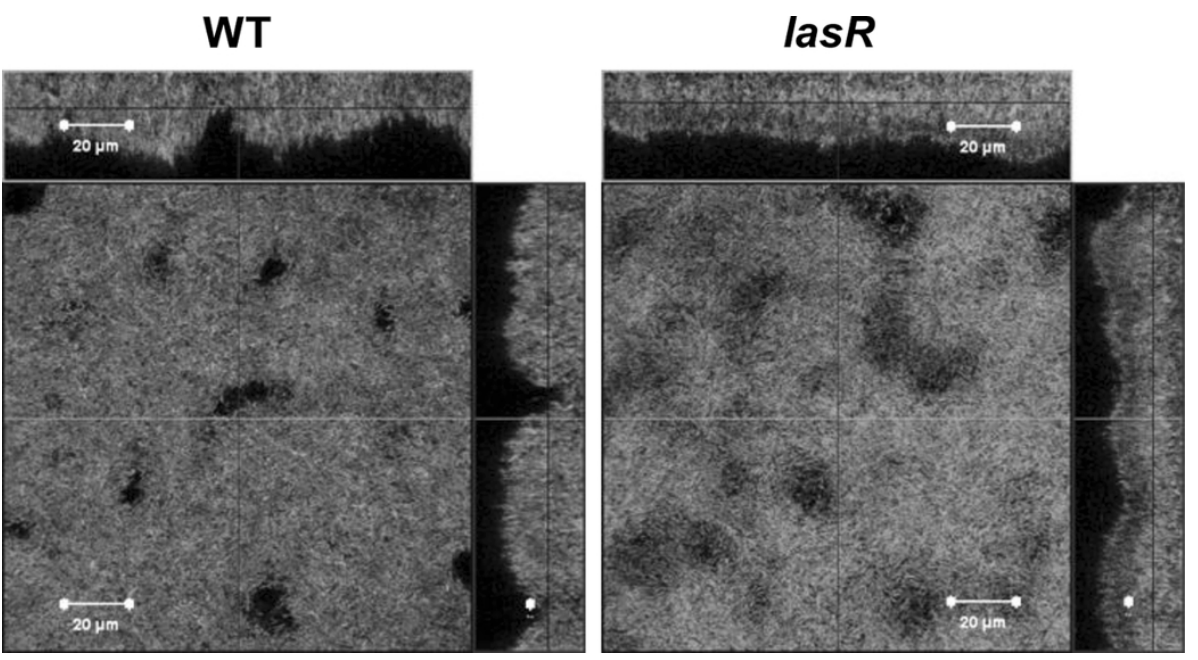

Figure 5 Flow-cell biofilms. CLSM images of flow-cell grown biofilms of the ZK wild-type (WT) and the lasR mutant at $37^{\circ} \mathrm{C}$ after 3 days. The large panel shows the horizontal cross-section and the small panel shows the vertical cross-section of the biofilm. The lines in the panels indicate the planes of the cross-sections.

las $R$ pqs $H$ double mutant in the ZK background. If a Series A congener caused wrinkling, then a las $R$ pqs $H$ mutant should still be wrinkly, and a $p q s H$ mutant would also be wrinkly if a Series A congener accumulates. Indeed, the degree of wrinkling generally correlated well with the amount of Series A congener produced, in the order lasR-pqsA::Tn $<\mathrm{WT}<p q s H$ $<$ lasR and lasR pqsH (Figure 8A). The wrinkly las $R$ mutant and the lasR pqsH double mutant produce the most, while the smooth wild-type produces considerably less (Figure $8 \mathrm{~B}$ ). The fact that the wrinkly las $\mathrm{R} p q s H$ mutant does not produce Series B congeners implies a role for a Series A congener. It is not clear why the pqs $H$ single mutant does not overproduce Series A congeners as previously shown for strain PA14 [27]. It is possible that $p q s A-E$ expression is reduced because indirect inhibition by the las system via the rhl system and the lack of the strong inducer PQS have a larger effect in the ZK background. Regardless, the partially wrinkly phenotype of the $p q s H$ mutant indicates that in addition to absolute abundance, the ratio of Series A to $B$ congeners may also be important. Densitometric analysis of wild-type and lasR mutant TLC spot intensities indeed shows that the Series A to Series B ratio is reciprocal in the two strains (Figure 8C).
Two Series A compounds, the PQS precursor HHQ and $\mathrm{HNQ}$, have been shown to be overproduced in a lasR mutant [20]. To examine whether one of these compounds is responsible for the wrinkly morphology of the lasR mutant, we added them to the lasR pqsA suppressor mutant. Exogenous addition to the agar medium or directly to the bacterial inoculum did not result in any change in colony morphology (data not shown). It is possible that diffusible AQ compounds are unable to enter cells in sufficient quantity, or that another less well-characterized Series A congener is responsible for the observed phenotype. Because exogenous complementation with diffusible $\mathrm{AQ}$ has been successful in the past $[60,61]$, we favor the latter.

\section{Conclusion}

In this study, we investigated the effect of las QS on biofilm formation and structure using a colony biofilm approach. This work was motivated by our recent global position analysis of LasR, which showed that this regulator directly binds to the $p s l$ polysaccharide promoter [8] (Figure 1). While we were unable to demonstrate the significance of this finding in the present study, we established a novel connection between las QS and the other major P. aeruginosa EPS, Pel. In particular, we

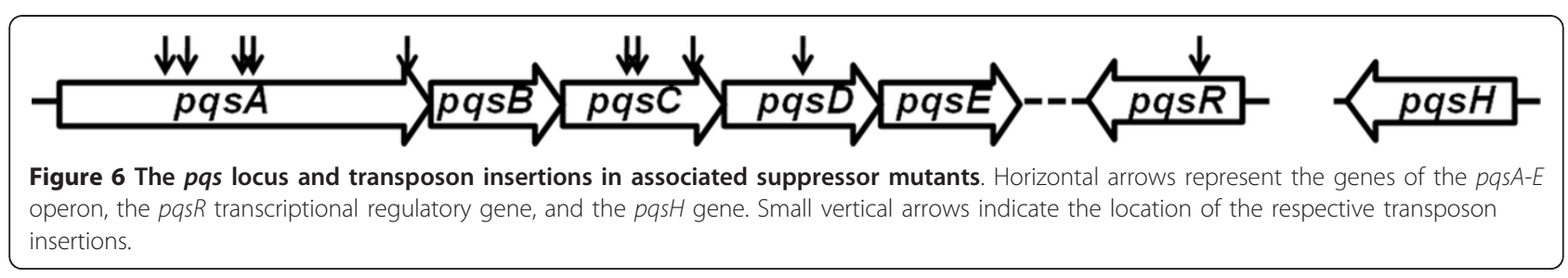




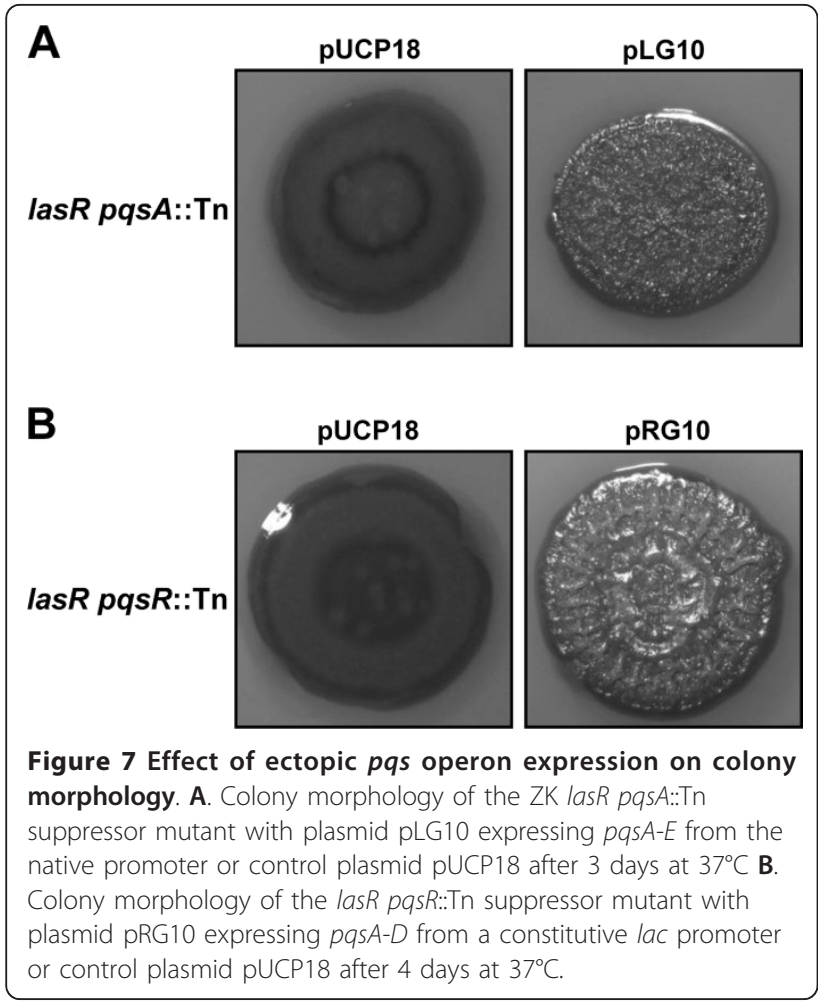

provide genetic evidence suggesting that the LasRI system represses Pel. We do not have any other independent evidence of this regulatory link as EPS composition analysis was unsuccessful. Las QS also only affected colonial morphology and did not affect biofilm formation in other relevant assays, including microtiter plate, pellicle, and flow-cell. It is conceivable that water availability (matric stress) is responsible for the conditionality of the observed phenotype.

It has previously been shown that LasRI induces Pel expression in strain PA14 at room temperature but not at $37^{\circ} \mathrm{C}$ [6]. This regulation is probably indirect and mediated via an unknown transcriptional regulator. Our finding that LasRI can also repress Pel expression in strain $\mathrm{ZK}$ at $37^{\circ} \mathrm{C}$, a temperature relevant to infection, raises the intriguing possibility that QS may trigger dissolution of clinical biofilms. This would be analogous to other bacterial pathogens like Vibrio cholerae [62] and Staphylococcus aureus [63]. Results with the particular strain chosen, ZK2870, are significant, because the autoaggregative behavior of this strain under some environmental conditions appears most representative among clinical and environmental isolates of $P$. aeruginosa [12]. The observed differences in the colony phenotype of different Pseudomonas strains (Figure 2) might be attributed to the presence or absence of a particular EPS locus or regulatory variability in strains with identical EPS loci.

Our second major finding is that las QS mediates colony morphology via AQ signaling. Phenotypic analysis along with AQ signal quantitation by TLC suggested that a Series A congener is involved. PqsA-D produce at least 8 different compounds within this
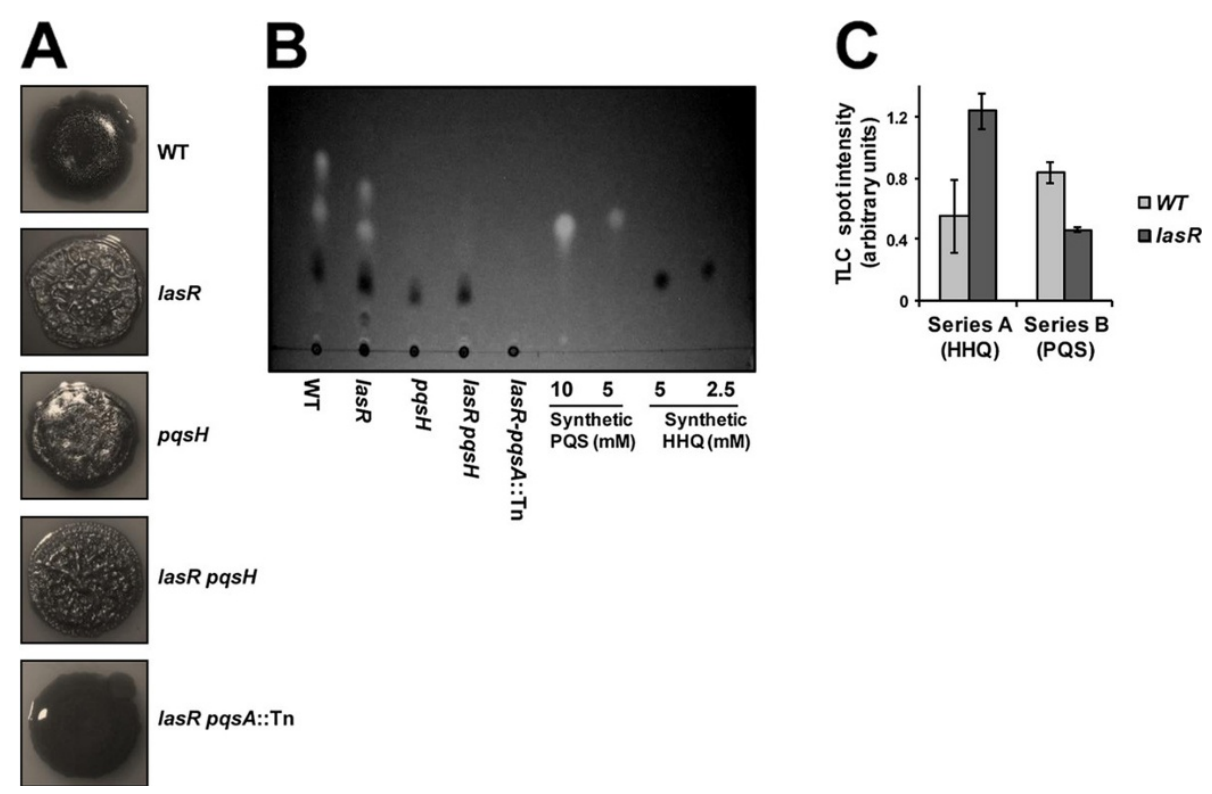

Figure 8 Colony morphology and AQ production of various QS mutants. A. Colony morphology of the ZK wild-type (WT), lasR, pqsH, las $R$ pqsH double mutant, and las R pqsA::Tn suppressor mutant after 5 days at $37^{\circ} \mathrm{C}$. B. TLC analysis of $\mathrm{AQ}$ production by the respective strains. Approximately $5 \mu \mathrm{l}$ of each sample (normalized to total amount of protein) was loaded. Note that samples towards the center of the plate ran more slowly than those near the edges. HHQ and PQS, representing Series A and B congeners, respectively, were included as synthetic controls. C. Densitometric analysis of TLC spot intensities in the wild-type and the lasR mutant from two independent experiments. 
series [64]. Of these, HHQ and HNQ have been shown to accumulate in a PAO1 lasR mutant [20]. Other prominent AQs, 2,4-dihydroxyquinoline (DHQ) and 2heptyl-4-hydroxyquinoline $N$-oxide (HQNO), that require some of the enzymes encoded by pqs $A-D$, but are not $\mathrm{PqsH}$ substrates, show reduced levels in a las $R$ mutant compared with the wild-type [20]. Our chemical supplementation experiments indicate that neither HHQ nor HNQ modulate wrinkling. This implies that one of the other less-well characterized Series A congeners have a role in this process, further expanding the various cellular functions of AQs in P. aeruginosa. A detailed investigation utilizing liquid chromatography/mass spectrometry along with chemical synthesis would be able to identify the compound in question. PqsE, a putative regulator encoded by the pqsA-E operon whose precise function is not known, is unlikely to have a role in AQ-mediated colony wrinkling, because $p q s A-D$ expression in a las $R$ pqs $R$ mutant that does not express $p q s E$ was sufficient to induce wrinkling (Figure 7B).

Interestingly, in Burkholderia pseudomallei the lack rather than the overproduction of the Series A congener HHQ results in a wrinkly colony phenotype [61]. In addition, AQ signal overproduction has previously been shown to induce autolysis in $P$. aeruginosa populations, forming plaques that result in characteristic translucent zones in colonies [36], different from those we observed. Autolysis appears to be mediated by PQS rather than a Series A congener [65].

Taken together, our data can be rationalized as follows: In the wild-type, both Series A and Series B congeners are produced as LasR activates $p q s R$ and $p q s H$. In the lasR mutant, Series A congeners accumulate and the Series A to Series B ratio increases because of (1) reduced $p q s H$ expression and (2) presumably las $R$-independent expression of pqs $R$ [25] resulting in continued activation of $p q s A-E$. In this case, one or more Series A congeners activate Pel production postranscriptionally in a PqsR-independent fashion, manifesting in a wrinkled phenotype. Colony morphology would be affected by a combination of pel-dependent and independent mechanisms, as lasR-mediated wrinkling was only partially pel-dependent (Figure 3). The particular AQ compound could alter colony morphology by binding to a novel receptor protein or through membrane interactions. While both PQS and HHQ have been shown to associate with outer membrane LPS, only PQS induces vesicle formation [66]. Such distinct interactions might have direct macroscopic effects on colony morphology, but might also alter the periplasmic environment in a way that affects the signaling status of receptor proteins in the cytoplasmic membrane. Posttranscriptional regulation of Pel could be mediated via a transmembrane signaling pathway that involves the LadS/RetS/GacS/ GacA two-component system, the RNA-binding protein RsmA and the small RNA RsmZ [67]. Pel translation has been shown to be repressed by the RNA-binding protein RsmA [68].

\section{Additional material}

\section{Additional file 1: Table S1. Oligonucleotides for deletion,}

overexpression, and reporter fusion constructs.

Additional file 2: Table S2. List of insertion mutants with the location

of the transposon insertion.

\section{Acknowledgements}

We thank Roberto Kolter for providing P. aeruginosa strain ZK2870 and pel, ps/ mutants, and we thank Colin Manoil for providing plasmid pLG10. We acknowledge Steve Diggle, Paul Williams and Marvin Whiteley for their kind gift of PQS, HNQ and HHQ signals, respectively. We also thank Matt Parsek and Kelly Colvin for their suggestions. This work was supported by NIH grant Al079454 and by start-up funds from Oregon State University (both to MS).

\section{Authors' contributions}

MS and RG designed and RG performed the experiments. RG and MS analyzed and interpreted the results. RG drafted the manuscript and MS critically revised it. All authors read and approved the final manuscript.

Received: 7 July 2011 Accepted: 9 March 2012 Published: 9 March 2012

\section{References}

1. Kerr KG, Snelling AM: Pseudomonas aeruginosa: a formidable and everpresent adversary. J Hosp Infect 2009, 73(4):338-344.

2. Fux CA, Costerton JW, Stewart PS, Stoodley P: Survival strategies of infectious biofilms. Trends Microbiol 2005, 13(1):34-40.

3. Branda SS, Vik S, Friedman L, Kolter R: Biofilms: the matrix revisited. Trends Microbiol 2005, 13(1):20-26.

4. Shapiro JA: The Use of Mudlac Transposons as Tools for Vital Staining to Visualize Clonal and Non-Clonal Patterns of Organization in BacterialGrowth on Agar Surfaces. J Gen Microbiol 1984, 130(1):1169-1181.

5. Hickman JW, Tifrea DF, Harwood CS: A chemosensory system that regulates biofilm formation through modulation of cyclic diguanylate levels. Proc Natl Acad Sci USA 2005, 102(40):14422-14427.

6. Sakuragi $Y$, Kolter R: Quorum-sensing regulation of the biofilm matrix genes (pel) of Pseudomonas aeruginosa. J Bacteriol 2007, 189(14):5383-5386.

7. Karatan E, Watnick P: Signals, regulatory networks, and materials that build and break bacterial biofilms. Microbiol Mol Biol Rev 2009, 73(2):310-347.

8. Gilbert KB, Kim TH, Gupta R, Greenberg EP, Schuster M: Global position analysis of the Pseudomonas aeruginosa quorum-sensing transcription factor LasR. Mol Microbiol 2009, 73(6):1072-1085.

9. Jackson KD, Starkey M, Kremer S, Parsek MR, Wozniak DJ: Identification of $p s l$, a locus encoding a potential exopolysaccharide that is essential for Pseudomonas aeruginosa PAO1 biofilm formation. J Bacteriol 2004, 186(14):4466-4475

10. Matsukawa M, Greenberg EP: Putative exopolysaccharide synthesis genes influence Pseudomonas aeruginosa biofilm development. J Bacteriol 2004, 186(14):4449-4456.

11. Friedman L, Kolter R: Genes involved in matrix formation in Pseudomonas aeruginosa PA14 biofilms. Mol Microbiol 2004, 51(2):675-690.

12. Friedman $L$, Kolter $R$ : Two genetic loci produce distinct carbohydrate-rich structural components of the Pseudomonas aeruginosa biofilm matrix. J Bacteriol 2004, 186(14):4457-4465.

13. Ma LY, Lu HP, Sprinkle A, Parsek MR, Wozniak DJ: Pseudomonas aeruginosa Psl is a galactose- and mannose-rich exopolysaccharide. J Bacterio/ 2007, 189(22):8353-8356 
14. Schuster M, Greenberg EP: A network of networks: quorum-sensing gene regulation in Pseudomonas aeruginosa. Int J Med Microbiol 2006, 296(23):73-81.

15. Juhas M, Eberl L, Tummler B: Quorum sensing: the power of cooperation in the world of Pseudomonas. Environ Microbiol 2005, 7(4):459-471.

16. Latifi A, Foglino M, Tanaka K, Williams P, Lazdunski A: A hierarchical quorum-sensing cascade in Pseudomonas aeruginosa links the transcriptional activators LasR and RhIR (VsmR) to expression of the stationary-phase sigma factor RpoS. Mol Microbiol 1996, 21(6):1137-1146.

17. Pesci EC, Pearson JP, Seed PC, Iglewski BH: Regulation of las and $r h l$ quorum sensing in Pseudomonas aeruginosa. J Bacteriol 1997, 179(10):3127-3132.

18. Diggle SP, Cornelis $P$, Williams $P$, Camara M: 4-quinolone signalling in Pseudomonas aeruginosa: old molecules, new perspectives. Int I Med Microbiol 2006, 296(2-3):83-91.

19. Heeb S, Fletcher MP, Chhabra SR, Diggle SP, Williams P, Camara M: Quinolones: from antibiotics to autoinducers. FEMS Microbiol Rev 2011, 35(2):247-274.

20. Deziel E, Lepine F, Milot S, He J, Mindrinos MN, Tompkins RG, Rahme LG: Analysis of Pseudomonas aeruginosa 4-hydroxy-2-alkylquinolines (HAQs) reveals a role for 4-hydroxy-2-heptylquinoline in cell-to-cell communication. Proc Natl Acad Sci USA 2004, 101(5):1339-1344.

21. Xiao GP, Deziel E, He JX, Lepine F, Lesic B, Castonguay MH, Milot S, Tampakaki AP, Stachel SE, Rahme LG: MvfR, a key Pseudomonas aeruginosa pathogenicity LTTR-class regulatory protein, has dual ligands. Mol Microbiol 2006, 62(6):1689-1699.

22. Wade DS, Calfee MW, Rocha ER, Ling EA, Engstrom E, Coleman JP, Pesci EC Regulation of Pseudomonas quinolone signal synthesis in Pseudomonas aeruginosa. J Bacterio/ 2005, 187(13):4372-4380.

23. Fletcher MP, Diggle SP, Camara M, Williams P: Biosensor-based assays for $\mathrm{PQS}, \mathrm{HHQ}$ and related 2-alkyl-4-quinolone quorum sensing signal molecules. Nat Protoc 2007, 2(5):1254-1262.

24. Gallagher LA, McKnight SL, Kuznetsova MS, Pesci EC, Manoil C: Functions required for extracellular quinolone signaling by Pseudomonas aeruginosa. J Bacteriol 2002, 184(23):6472-6480.

25. Diggle SP, Winzer K, Chhabra SR, Worrall KE, Camara M, Williams P: The Pseudomonas aeruginosa quinolone signal molecule overcomes the cell density-dependency of the quorum sensing hierarchy, regulates $r \mathrm{hl}$ dependent genes at the onset of stationary phase and can be produced in the absence of LasR. Mol Microbiol 2003, 50(1):29-43.

26. McGrath $S$, Wade DS, Pesci EC: Dueling quorum sensing systems in Pseudomonas aeruginosa control the production of the Pseudomonas quinolone signal (PQS). Fems Microbiol Lett 2004, 230(1):27-34.

27. Xiao G, He J, Rahme LG: Mutation analysis of the Pseudomonas aeruginosa mvfR and pqSABCD gene promoters demonstrates complex quorum-sensing circuitry. Microbiology 2006, 152(Pt 6):1679-1686.

28. Lepine F, Dekimpe V, Lesic B, Milot $S$, Lesimple A, Mamer OA, Rahme LG, Deziel E: PqsA is required for the biosynthesis of 2,4-dihydroxyquinoline (DHQ), a newly identified metabolite produced by Pseudomonas aeruginosa and Burkholderia thailandensis. Biol Chem 2007, 388(8):839-845.

29. Hentzer M, Wu H, Andersen JB, Riedel K, Rasmussen TB, Bagge N, Kumar N, Schembri MA, Song Z, Kristoffersen P, et al: Attenuation of Pseudomonas aeruginosa virulence by quorum sensing inhibitors. EMBO J 2003, 22(15):3803-3815.

30. Deziel E, Gopalan S, Tampakaki AP, Lepine F, Padfield KE, Saucier M, Xiao G, Rahme LG: The contribution of MvfR to Pseudomonas aeruginosa pathogenesis and quorum sensing circuitry regulation: multiple quorum sensing-regulated genes are modulated without affecting las $R$, rhIR or the production of N-acyl-L-homoserine lactones. Mol Microbiol 2005, 55(4):998-1014.

31. Schuster M, Lostroh CP, Ogi T, Greenberg EP: Identification, timing, and signal specificity of Pseudomonas aeruginosa quorum-controlled genes: a transcriptome analysis. J Bacteriol 2003, 185(7):2066-2079.

32. Wagner VE, Bushnell D, Passador L, Brooks Al, Iglewski BH: Microarray analysis of Pseudomonas aeruginosa quorum-sensing regulons: effects of growth phase and environment. J Bacteriol 2003, 185(7):2080-2095.

33. Davies DG, Parsek MR, Pearson JP, Iglewski BH, Costerton JW, Greenberg EP: The involvement of cell-to-cell signals in the development of a bacterial biofilm. Science 1998, 280(5361):295-298.

34. Ueda A, Wood TK: Connecting quorum sensing, c-di-GMP, pel polysaccharide, and biofilm formation in Pseudomonas aeruginosa through tyrosine phosphatase TpbA (PA3885). PLoS Pathog 2009, 5(6): e1000483.

35. Davey ME, Caiazza NC, OToole GA: Rhamnolipid surfactant production affects biofilm architecture in Pseudomonas aeruginosa PAO1. J Bacteriol 2003, 185(2):1027-1036.

36. D'Argenio DA, Calfee MW, Rainey PB, Pesci EC: Autolysis and autoaggregation in Pseudomonas aeruginosa colony morphology mutants. J Bacteriol 2002, 184(23):6481-6489.

37. Allesen-Holm M, Barken KB, Yang L, Klausen M, Webb JS, Kjelleberg S, Molin S, Givskov M, Tolker-Nielsen T: A characterization of DNA release in Pseudomonas aeruginosa cultures and biofilms. Mol Microbiol 2006, 59(4):1114-1128

38. Shrout JD, Chopp DL, Just CL, Hentzer M, Givskov M, Parsek MR: The impact of quorum sensing and swarming motility on Pseudomonas aeruginosa biofilm formation is nutritionally conditional. Mol Microbiol 2006, 62(5):1264-1277.

39. Rahme LG, Stevens EJ, Wolfort SF, Shao J, Tompkins RG, Ausubel FM: Common virulence factors for bacterial pathogenicity in plants and animals. Science 1995, 268(5219):1899-1902.

40. Holloway BW, Krishnapillai V, Morgan AF: Chromosomal genetics of Pseudomonas. Microbiol Rev 1979, 43(1):73-102.

41. Wilder CN, Diggle SP, Schuster M: Cooperation and cheating in Pseudomonas aeruginosa: the roles of the las, rhl and pqs quorumsensing systems. ISME J 2011, 5(8):1332-1343.

42. Liberati NT, Urbach JM, Miyata S, Lee DG, Drenkard E, Wu G, Villanueva J, Wei T, Ausubel FM: An ordered, nonredundant library of Pseudomonas aeruginosa strain PA14 transposon insertion mutants. Proc Natl Acad SC USA 2006, 103(8):2833-2838.

43. Simon R, UPAP : A Broad Host Range Mobilization System for In Vivo Genetic Engineering: Transposon Mutagenesis in Gram Negative Bacteria. Nat Biotech 1983, 1:784-791.

44. Becher A, Schweizer HP: Integration-proficient Pseudomonas aeruginosa vectors for isolation of single-copy chromosomal lacZ and lux gene fusions. Biotechniques 2000, 29(5):948-950-952.

45. Hoang TT, Karkhoff-Schweizer RR, Kutchma AJ, Schweizer HP: A broad-hostrange Flp-FRT recombination system for site-specific excision of chromosomally-located DNA sequences: application for isolation of unmarked Pseudomonas aeruginosa mutants. Gene 1998, 212(1):77-86.

46. Heeb S, Blumer C, Haas D: Regulatory RNA as mediator in GacA/RsmAdependent global control of exoproduct formation in Pseudomonas fluorescens CHA0. J Bacteriol 2002, 184(4):1046-1056.

47. Schweizer HP: Escherichia-Pseudomonas shuttle vectors derived from pUC18/19. Gene 1991, 97(1):109-121.

48. Horton RM, Cai ZL, Ho SN, Pease LR: Gene splicing by overlap extension: tailor-made genes using the polymerase chain reaction. Biotechniques 1990, 8(5):528-535.

49. Bradford MM: A rapid and sensitive method for the quantitation of microgram quantities of protein utilizing the principle of protein-dye binding. Anal Biochem 1976, 72:248-254.

50. Whiteley M, Lee KM, Greenberg EP: Identification of genes controlled by quorum sensing in Pseudomonas aeruginosa. Proc Natl Acad Sci USA 1999, 96(24):13904-13909.

51. Coic R, Kowalik T, Quarles JM, Stevenson B, K TR: Growing and analyzing biofilms in flow-cells. In Current Protocols in Microbiology. Volume 1. Wiley and Sons Inc; New Jersey; 2006.

52. Fox A, Haas D, Reimmann C, Heeb S, Filloux A, Voulhoux R: Emergence of secretion-defective sublines of Pseudomonas aeruginosa PAO1 resulting from spontaneous mutations in the vfr global regulatory gene. Appl Environ Microbiol 2008, 74(6):1902-1908.

53. Larsen RA, Wilson MM, Guss AM, Metcalf WW: Genetic analysis of pigment biosynthesis in Xanthobacter autotrophicus Py2 using a new, highly efficient transposon mutagenesis system that is functional in a wide variety of bacteria. Arch Microbiol 2002, 178(2):193-201.

54. Spiers AJ, Bohannon J, Gehrig SM, Rainey PB: Biofilm formation at the airliquid interface by the Pseudomonas fluorescens SBW25 wrinkly spreader requires an acetylated form of cellulose. Mol Microbiol 2003, 50(1):15-27.

55. Dietrich LE, Teal TK, Price-Whelan A, Newman DK: Redox-active antibiotics control gene expression and community behavior in divergent bacteria. Science 2008, 321(5893):1203-1206.

56. Colvin KM, Gordon VD, Murakami K, Borlee BR, Wozniak DJ, Wong GCL, Parsek MR: The Pel polysaccharide can serve a structural and protective 
role in the biofilm matrix of Pseudomonas aeruginosa. Plos Pathog 2011, 7(1):e1001264.

57. Chang WS, Halverson LJ: Reduced water availability influences the dynamics, development, and ultrastructural properties of Pseudomonas putida biofilms. J Bacteriol 2003, 185(20):6199-6204.

58. Rampioni G, Pustelny C, Fletcher MP, Wright VJ, Bruce M, Rumbaugh KP, Heeb S, Camara M, Williams P: Transcriptomic analysis reveals a global alkyl-quinolone-independent regulatory role for PqsE in facilitating the environmental adaptation of Pseudomonas aeruginosa to plant and animal hosts. Environ Microbiol 2010, 12(6):1659-1673.

59. D'Argenio DA, Wu M, Hoffman LR, Kulasekara HD, Deziel E, Smith EE, Nguyen H, Ernst RK, Larson Freeman TJ, Spencer DH, et al: Growth phenotypes of Pseudomonas aeruginosa lasR mutants adapted to the airways of cystic fibrosis patients. Mol Microbiol 2007, 64(2):512-533.

60. Ha DG, Merritt JH, Hampton TH, Hodgkinson JT, Janecek M, Spring DR, Welch M, OToole GA: 2-Heptyl-4-Quinolone, a Precursor of the Pseudomonas Quinolone Signal Molecule, Modulates Swarming Motility in Pseudomonas aeruginosa. J Bacteriol 2011, 193(23):6770-6780.

61. Diggle SP, Lumjiaktase P, Dipilato F, Winzer K, Kunakorn M, Barrett DA, Chhabra SR, Camara M, Williams P: Functional genetic analysis reveals a 2Alkyl-4-quinolone signaling system in the human pathogen Burkholderia pseudomallei and related bacteria. Chem Biol 2006, 13(7):701-710.

62. Hammer BK, Bassler BL: Quorum sensing controls biofilm formation in Vibrio cholera. Mol Microbiol 2003, 50(1):101-104.

63. Boles BR, Horswill AR: Agr-mediated dispersal of Staphylococcus aureus biofilms. PLoS Pathog 2008, 4(4):e1000052.

64. Lepine F, Milot S, Deziel E, He JX, Rahme LG: Electrospray/mass spectrometric identification and analysis of 4-hydroxy-2-alkylquinolines (HAQs) produced by Pseudomonas aeruginosa. J Am Soc Mass Spectr 2004, 15(6):862-869.

65. Haussler S, Becker T: The Pseudomonas quinolone signal (PQS) balances life and death in Pseudomonas aeruginosa populations. PLoS Pathog 2008, 4(9):e1000166.

66. Mashburn-Warren L, Howe J, Garidel P, Richter W, Steiniger F, Roessle M, Brandenburg K, Whiteley M: Interaction of quorum signals with outer membrane lipids: insights into prokaryotic membrane vesicle formation. Mol Microbiol 2008, 69(2):491-502

67. Ventre I, Goodman AL, Vallet-Gely I, Vasseur P, Soscia C, Molin S, Bleves S, Lazdunski A, Lory S, Filloux A: Multiple sensors control reciprocal expression of Pseudomonas aeruginosa regulatory RNA and virulence genes. Proc Natl Acad Sci USA 2006, 103(1):171-176.

68. Brencic A, Lory S: Determination of the regulon and identification of novel mRNA targets of Pseudomonas aeruginosa RsmA. Mol Microbiol 2009, 72(2):612-632.

doi:10.1186/1471-2180-12-30

Cite this article as: Gupta and Schuster: Quorum sensing modulates colony morphology through alkyl quinolones in Pseudomonas aeruginosa. BMC Microbiology 2012 12:30.

\section{Submit your next manuscript to BioMed Central and take full advantage of:}

- Convenient online submission

- Thorough peer review

- No space constraints or color figure charges

- Immediate publication on acceptance

- Inclusion in PubMed, CAS, Scopus and Google Scholar

- Research which is freely available for redistribution

Submit your manuscript at www.biomedcentral.com/submit
Biomed Central 